\title{
Research and Design of Ballistic Missile Against Large Ship Simulation
}

\section{System}

\author{
YAO Qun, SHU Jian-sheng, ZHANG Bin-wei
}

The Rocket Force University of Engineering, Xi'an 710025, China

\begin{abstract}
Abatract: Complied with HLA specification based on MAK- RTI software for run support, the highly stable and extend-able ballistic missile against large ship simulation system is designed and implemented. The system simulated formation of large ship, satellite recon, command of battle and the process of missile attacking large ship. The paper introduces the related key technologies and its realization in the process of developing the system.
\end{abstract}

Keywords: HLA, ballistic missile, formation of large ship

\section{Introduction}

The aircraft carrier is a large surface ship that carries the carrier-based fighter as its main task, aircraft carrier battle group is the aircraft carrier led the fleet, which owned by the naval forces. The carrier battle group combines the efficient transport of the ship with the sharp mobility of the fighter, the sea and air force coordination capability enhanced greatly, the ability of the sea, air and long-range troops to quickly deliver improved, and increasingly become an important deterrent force at sea.

While China's long coastline is a large ocean, involving more disputes over maritime rights and interests. But China started late in the aircraft carrier R \& D and manufacturing, and the naval warfare capabilities do not occupy an absolute advantage in the same conditions. In order to safeguard maritime rights and homeland security, to ensure that maritime traffic and territorial waters are not subject to foreign aircraft and other large maritime forces violations. While vigorously developing the naval aircraft carrier strength construction, it should also accelerate the development of the aircraft carrier battle group has a strong threat to the ballistic missile air-defense ship operations, and the formation of a strong coastal protection capabilities.

In the attack on the aircraft carrier formation, the attack side of the combat platform must reconcile the mobile target positioning at the first time, the implementation of the launch must be done after obtaining specific target information. The missile will face the interception of the aircraft carrier battlefield anti-missile system after the successful launch. In other words, the missile can successfully hit the target and complete the combat aircraft carrier battle mission just under the premise of a successful penetration [ 1-3 ].

This paper mainly introduces the function and composition of a design of battle back combat system for ballistic missile based on the HLA framework, and discusses some key technologies and implementation ways of constructing the simulation platform, which is helpful for the design and development of similar simulation system. 


\section{System design}

\subsection{System Structure}

Ballistic missile combat large-scale ship simulation system is an offensive and defensive simulation model which mainly used to study the ballistic missile forces under the support of the marine surveillance satellite to break through the aircraft carrier fleet defense system and directly damage the aircraft carrier. Based on HLA technology, running support software using MAK-RTI, the system consists of red and blue sides, the blue side according to the combat plan to create aircraft carrier formation for aircraft carrier formation. The red side of the ocean surveillance satellite system is used to detect the location information of the aircraft carrier and provide the target parameters for the red missile weapon. The red command and control center is used to deal with the target information of the aircraft carrier, deploy the combat task, carry out the command and decision for the anti- Red missile used to attack the blue side of the aircraft carrier target. At the same time, the blue side has early warning satellite members to predict the red missile parameters which provided to the aircraft carrier control center for the aircraft carrier anti-missile service. Its composition is shown in Fig. Its composition structure shown in Fig.1.

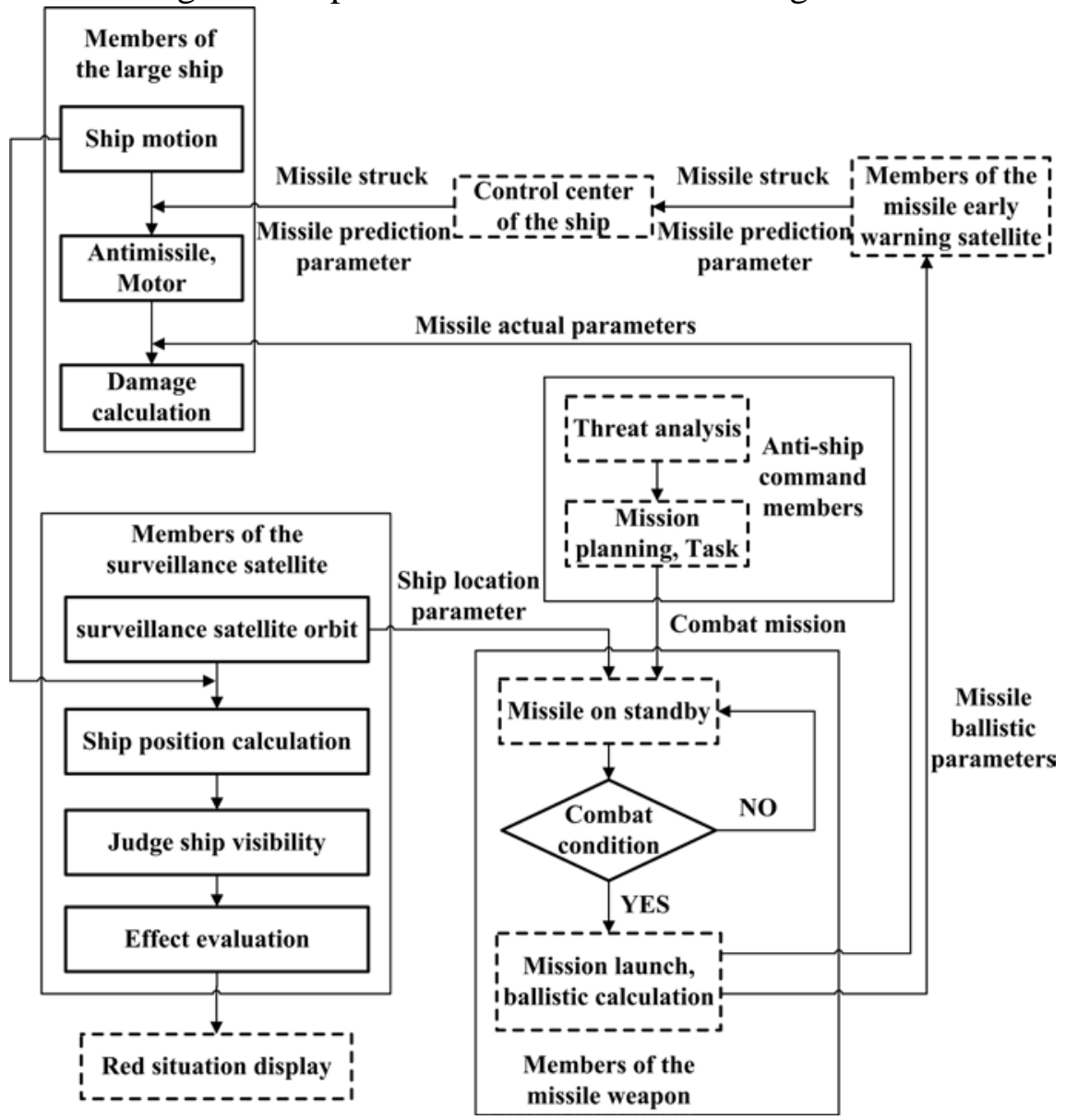

Fig.1 Information process of ballistic missile against large ship simulation system

\subsection{Architecture}

According to the simulation process of ballistic missile anti-aircraft carrier combat simulation system, using the advantages of HLA, mainly in the application layer design and development, designed the various members of the federal object class and interactive class. According to the 
simulation system contains the model, model function, the relationship between the model and the missile attack large ship process, the simulation system is divided into six categories and a large module:

(1)Large ship formation battle group strength simulation members. The main function is based on the initial data to create the aircraft carrier formation object, the aircraft carrier formation simulation, the aircraft carrier damage level calculation and release.

(2)Missile warning satellite simulation members.The main function is to search the missile target for missile warning early warning range, to establish the missile weapon target object class, to deal with the missile weapon object according to the calculation and delay model of the missile early warning satellite, and to release the missile weapon object attribute.

(3) Large Naval Fleet command and control simulation center members.The main function is to receive the missile target from the missile early warning satellite, carry out the threat analysis and mission planning, and issue the missile attack to the aircraft carrier formation members.

(4) Marine surveillance satellite simulation members.The main function is to order the aircraft carrier formation object class, the aircraft carrier position state calculation, and release the target object class.

(5) Combat large-scale ship command and control simulation members.The main function is to order the reconnaissance satellite simulation members issued by the aircraft carrier target information, threat analysis and mission planning, issued missile weapons attack aircraft carrier combat missions.

(6)Missile weapon simulation members.The main function is to create the missile object, the missile trajectory calculation and display, and release the missile's position and speed.

At the same time, the connection of six categories of members, and ultimately to achieve the simulation run also need to combat process and battlefield situation to establish a simulation platform, the basic functions: Simulation of ballistic missile forces assault aircraft carrier battle against the two sides of the main combat operations and combat process, simulation by capturing ballistic missiles to burn the tail of the infrared radiation to detect the ballistic missile launch and tracking identification, demonstration and evaluation of the missile forces assault aircraft carrier battle tactics and programs, dynamic display of missile forces assault aircraft carrier battle effect and the occurrence of real-time events, the use of space-based information system to achieve the aircraft carrier fleet of large-scale moving targets such as reconnaissance Positioning capabilities and training systems for integrating higher levels of training. The basic requirements of its performance include four points: First, the system is stable, reliable and repeatability, scalability and strong; Second, the description of the simulation model to have a certain degree of accuracy; Third, the command and control activities, systems Simulation process control and other artificial intervention sensitive; Fourth, a wide range of moving target monitoring and positioning with high efficiency, hidden and safe features[ 4 ].

\section{System Development}

\subsection{Development Process}

To develop an interactive simulation system based on HLA, we should clarify the composition of federal members and federations and determine the interaction and interaction between federates. To establish the FOM and SOM, and then model the implementation of each federated member. In order to improve the efficiency of simulation system development, the US DMSO (Department of 
Defense Modeling and Simulation Office) proposed FEDEP(Federation Development Execute Modal). The model describes all the necessary activities and processes around federated development, as well as a detailed checklist for each step. Although the development process for each simulation system is different, the most basic process is the same. FEDEP abstracts the federal development process into six basic steps: The first step is to define the federal goal, that is, to define the objectives of federal development; The second step is to develop a federal conceptual model that is designed according to federal development goals; The third step is to design the federation, which is based on the design of federal members; The fourth step is to develop a federation, the purpose of which is to develop FOM; The fifth step is to synthesize and test the federation, the purpose of which is to examine and test whether the federated object model achieves the simulation goal; The sixth step is to perform federal and analytical results.

The content of the federated member model mainly includes the federal member entity model, the interaction model and the simulation loop body. The solid model directly describes the behavioral characteristics of the entity itself, either a set of dynamics and kinematics equations, or a set of event flows that can be used to establish a solid model using traditional continuous system and discrete event system simulation methods. The interaction model describes the interaction between entities in the system, based on OMT's FOM and SOM tables. The simulation loop is a concrete realization of the simulation of the entity. These three parts are organically combined to form a federated member to accomplish a specific simulation task.

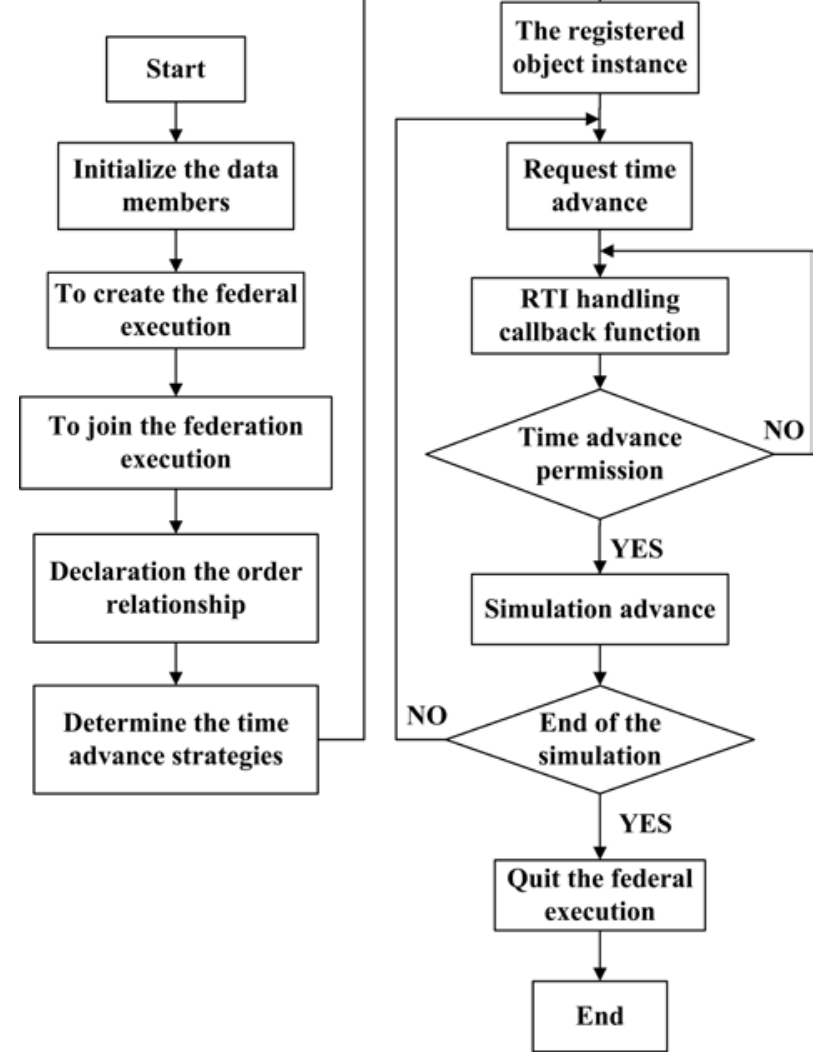

Fig.2 Federal member implementation process

Federal member implementation process shown in Fig.2, The entire lifecycle of the federal execution is managed and maintained by RTI, which is done by the creation / revocation of the federation implementation, the joining / exiting of the member, and the registration / deletion of the object instance, all based on the request of the federated member. For each federated member, the steps and operations shown in Figure 3 are similar. Therefore, the development of a common HLA 
membership framework to complete these tasks, the basic process package, to further reduce the workload of the preparation of member code to improve the efficiency of federal development.

\subsection{Software Integration}

The system development environment using Microsoft's VC ++ integrated development framework, the third party software integration is the use of $\mathrm{C}++$ and commercial software interface, unified integration in the Visual $\mathrm{C}++$ environment. In the missile weapon combat members and early warning satellite simulation members, the use of the United States Analytical Graphics company developed STK satellite tool software for comprehensive analysis and display. In the STK in the dynamic generation of multiple missiles, and show the head of the separation of ground explosion and other renderings, simulation early warning satellite tracking missile launch process, with a number of two-dimensional map, three-dimensional map from different angles on the simulation process in all directions Display, for the simulation management and control members to provide decision-making basis. The STK / Connect module provides the ability of the user to connect to the STK in the client / server environment, providing a communication tool for other applications to send messages to the STK and receive data from the STK. Connect can selectively generate information, users can also cancel the standard information, use a custom format to adapt to the application. This feature allows developers to better control the information environment. Members join the federation, start the STK process, establish a connection with the STK, according to the simulation management node instructions to load the appropriate scene, start STK Animation, simulation is completed, uninstall STK scene[ 5 ].

\section{Key Technologies}

\subsection{Dead Reckoning Method}

DR (Dead Reckoning) algorithm is used in DIS protocol to reduce the physical location, speed PDU transmission frequency of the method, its expansion and introduction into the HLA, can reduce the amount of network data, improve the network bandwidth effect. The basic idea of DR algorithm in HLA is that the published member and the ordering member of the object class attribute maintain the same entity simplification model. The model does not take into account the external influence of the simulation entity, and only uses the simplified integral equation to carry out its own state the recursive solution. In the simulation process, the object class attribute publication member compares the simulation model and simplifies the attribute value calculation of the model, and the publication member invokes the update Attribute Values service only if the difference between them exceeds the pre-set threshold value. The DR algorithm is used to reduce the frequency of data transmission of object instance data, reduce network transmission delay and improve simulation efficiency. However, the DR algorithm adds the computational complexity of the entity simplification model to the member, and the more complex the detail of the entity simplifies the model, the lower the frequency of the object class attribute data transmission (the simplified model calculates the attribute value from the "true" value) The larger the model calculation. Therefore, DR algorithm in the role of improving the simulation of the existence of their own contradictory, in the system application without receiving a new entity state, the use of extrapolation method to predict changes in the physical location of the scene to refresh the scene to eliminate physical movement Of the discontinuous phenomenon. 


\subsection{Real-time Simulation of Step-size}

HLA use the TSO (Time Stamp Order) messages solve the problem that the events in the simulation world do not occur in sequence, but increases the difficulty of developing a real-time distributed interactive simulation system because the TSO message hinders the time limited time for members to advance. The fully coupled federated design allows the entire federal time to propel the speed depends on the most time-consuming members of the run. This is far from enough for HLA real-time simulation. If there is a large amount of computational complexity and difficult to parallelize a member, the time lag of the member / federation is largely unresolved.

The basic idea of the step-by-step method is to determine the step size of the time by calculating the run time of the member and to advance the state of the simulation model to the moment when the simulation is advanced. This solves the problem of member's response to external event response time, so that members no longer need time compensation during operation. For variable step-by-step real-time simulations, it is desirable to be able to deliver both TSO messages in the federation and to enable each member to achieve almost no effect by other members when making time advance. It has been found that this requirement can be met by setting the prospective amount of each member reasonably. It has been proved that in the variable-step real-time federation, each member satisfies the minimum amount required for its current simulation step. In the specific implementation process, you can set the current step forward amount of the first few simulation steps of the average. In addition, members in the simulation after the start of the first simulation step, in order not to affect the speed of advance, then the need to set the members of the prospective large enough. This will not affect the Fed's emulation function, because the impact of the prospective amount on the simulation function is only reflected in the reception delay of the TSO message, and in general the member does not generate significant TSO messages in the first simulation step.

Developed a variable step real time federated A for testing, with three members A1、A2 and A3, calculated time consuming for $0.5 \mathrm{~s}, 1.0 \mathrm{~s}$ and $1.5 \mathrm{~s}$ respectively. Among them, A1 is responsible for federal management, Between A2 and A3, there is bidirectional transmission of object attribute data and unidirectional transmission of interaction. After the start of the simulation, the forward size of the first step is set to 5.0s, and the amount of forwards is set to the simulation step size for the previous step. The hardware environment of the experiment is: 3 sets of the same PC, 100M bandwidth of the local area network; software environment: Windows 7 version, MAK- RTI / VR-LINK, Visual C ++ 6.0. At the same time, a fully coupled real-time federation A' corresponding to $\mathrm{A}$ is developed. The corresponding members are A1', A2'and A3' respectively, and the simulation step is set to 2.0s. And the development of a federal A, which differs from A in that the LRC thread and the federated member thread are independent, and the corresponding federated members are A1", A2" and A3 "respectively. The results of their tests were shown in Table.1. 
Table 1 Test result

\begin{tabular}{cccccc}
\hline Federal & $\begin{array}{c}\text { Federal } \\
\text { members }\end{array}$ & $\begin{array}{c}\text { Relative } \\
\text { error of } \\
\text { real-time/\% }\end{array}$ & $\begin{array}{c}\text { Simulation } \\
\text { resolution/s }\end{array}$ & $\begin{array}{c}\text { Time } \\
\text { utilization/\% }\end{array}$ & $\begin{array}{c}\text { Interactive } \\
\text { receiving } \\
\text { delay/s }\end{array}$ \\
\hline \multirow{3}{*}{$\mathrm{A}$} & $\mathrm{A}_{1}$ & 0.1319 & 0.5687 & 98.88 & - \\
& $\mathrm{A}_{2}$ & 0.1058 & 1.1546 & 98.45 & 0.5784 \\
& $\mathrm{~A}_{3}$ & 0.1746 & 1.5687 & 96.52 & - \\
\hline \multirow{3}{*}{$\mathrm{A}^{\prime}$} & $\mathrm{A}_{1}{ }^{\prime}$ & 0.0356 & 2.0000 & 24.59 & - \\
& $\mathrm{A}_{2}{ }^{\prime}$ & 0.0063 & 2.0000 & 54.23 & 1.0638 \\
& $\mathrm{~A}_{3}{ }^{\prime}$ & 0.0027 & 2.0000 & 74.37 & - \\
\hline \multirow{2}{*}{$\mathrm{A}^{\prime \prime}$} & $\mathrm{A}_{1}{ }^{\prime \prime}$ & 0.8541 & 0.6452 & 96.74 & - \\
& $\mathrm{A}_{2}{ }^{\prime \prime}$ & 0.5217 & 0.9875 & 97.56 & 0.0156 \\
& $\mathrm{~A}_{3}{ }^{\prime \prime}$ & 0.4388 & 1.8574 & 98.34 & - \\
\hline
\end{tabular}

It can be seen from the test results that, in addition to the relative real-time error, $\mathrm{A}$ is significantly better than $\mathrm{A}^{\prime}$ on other indicators, which indicates that the real-time federation is far superior to the fully coupled real-time federation in terms of resource utilization and simulation efficiency. A" greatly improves the state delay, but A" in other indicators are inferior to A', especially the relative real-time error is too large, so multiple threads on the computer resources the negative impact of snatching on real-time simulation is greater.

\section{Conclusions}

Because of the particularity of space warfare, simulation technology is an effective means to study space warfare. HLA-based distributed interactive simulation system has the advantages of high interoperability and high reusability, and represents the development direction of advanced simulation technology. This paper analyzes the object model and the information interaction mechanism of the attack and defense process by using the object - oriented method to follow the HLA - based framework and the rules of the HLA - based design scheme. The DR algorithm is used to improve the simulation efficiency. Real-time performance of the system is improved by real-time simulation with variable step size. The results of the system demonstrate the effectiveness of these methods. Due to the emphasis on the composition of the system and the key technology, some of the details will be simplified. The development of this system has a good reference value for the development of large complex simulation system.

\section{References}

[1] Zhou Yan, Dai Jian-wei. HLA policy program design [M]. Electronic Industry Press, 2012, 12-21.

[2] HAO Jiang-Bo, QING Du-Zheng. Study on the Development of Distributed Interactive Simulation System Based on HLA [J] .Journal of System Simulation, 2013, 24(1):50-53.

[3] Di Jian-wei, Jiang Xiao-yuan. Study on the Development of Weapon Countermeasure Simulation System Based on HLA [J]. Computer Simulation, 2014, 6(3):13-20.

[4] U Peng, QIU Da-shan. Experimental Simulation System of Joint Campaign [J]. Computer Simulation, 2015, 5(7):32-37.

[5] Liao Ying, Liang Jia-hong. Real-time simulation theory and support technology [M]. National Defense University of Science and Technology Press, 2012, 56-60. 